\begin{tabular}{|c|l|}
\hline Title & Slow switching in globally coupled oscillators: robustness and occurrence through delayed coupling \\
\hline Author(s) & Kori, Hiroshi; Kuramoto, Y oshiki \\
\hline Citation & $\begin{array}{l}\text { Physical Review E, 63(4), 046214 } \\
\text { https://doi.org/10.1103/PhysRevE.63.046214 }\end{array}$ \\
\hline Issue Date & 2001-03-29 \\
\hline Doc URL & http://hdl.handle.net/2115/17207 \\
\hline Rights & Copyright $\odot 2001$ American Physical Society \\
\hline Type & article \\
\hline File Information & PRE63-046214.pdf \\
\hline
\end{tabular}

Instructions for use 


\title{
Slow switching in globally coupled oscillators: robustness and occurrence through delayed coupling
}

\author{
Hiroshi Kori* and Yoshiki Kuramoto \\ Department of Physics, Graduate School of Sciences, Kyoto University, Kyoto 606-8502, Japan
}

(Received 19 September 2000; published 29 March 2001)

\begin{abstract}
The phenomenon of slow switching in populations of globally coupled oscillators is discussed. This characteristic collective dynamics, which was first discovered in a particular class of the phase oscillator model, is a result of the formation of a heteroclinic loop connecting a pair of clustered states of the population. We argue that the same behavior can arise in a wider class of oscillator models with the amplitude degree of freedom. We also argue how such heteroclinic loops arise inevitably and persist robustly in a homogeneous population of globally coupled oscillators. Although a heteroclinic loop might seem to arise only exceptionally, we find that it appears rather easily by introducing time delay into a population which would otherwise exhibit perfect phase synchrony. We argue that the appearance of the heteroclinic loop induced by the delayed coupling is then characterized by transcritical and saddle-node bifurcations. Slow switching arises when a system with a heteroclinic loop is weakly perturbed. This will be demonstrated with a vector model by applying weak noises. Other types of weak symmetry-breaking perturbations can also cause slow switching.
\end{abstract}

DOI: $10.1103 /$ PhysRevE.63.046214

PACS number(s): 05.45.Xt, 87.10.+e

\section{INTRODUCTION}

Coupled limit-cycle oscillators appear in various contexts in physics [1-4], chemistry [5,6], and biology [7-9]. Various types of collective behavior, which arise when they form large assemblies, have been studied extensively over the last few decades. Among the possible types of behavior, we will particularly be concerned with clustering and slow switching, which was first studied by Hansel et al. [10] in a homogeneous population of globally coupled phase oscillators. Assuming a simple form for the coupling function, they showed numerically that after a long transient the system approaches a two-cluster state, i.e., the whole population splits into two rigidly rotating subpopulations, each in perfect phase synchrony. However, the stability analysis of this two-cluster state revealed that it is linearly unstable, corresponding to a saddle point if seen in a corotating frame of reference. The seeming contradiction here was interpreted in terms of the formation of a heteroclinic loop connecting this two-cluster state and another two-cluster state which was obtained simply by a constant phase shift of the former. In fact, when this heteroclinic loop is attracting, the trajectory stays longer and longer near these saddle points, so that the numerical roundoff error finally forces the system to stay at one of the saddles forever. This interpretation was supported by the fact that when small external noise is included the system is no longer fixed at a saddle point but starts to repeat slow switchings between the pair of saddles (see Fig. 1). Although these findings are important, explanations still needed as to why the heteroclinic loop arises inevitably and persists robustly against our common belief in its structural instability.

In the next two sections, we restrict our consideration to the phase model. In Sec. II, we discuss in some detail the stability condition of the two-cluster state. Existing numeri-

\footnotetext{
*Email address: kori@ton.scphys.kyoto-u.ac.jp
}

cal results obtained by a particular model suggest the appearance of heteroclinic loops. Thus, in Sec. III, we argue the mechanism by which heteroclinic loops are necessarily formed. Specifically, a sufficient condition will be given for the existence of a heteroclinic loop, and how this condition is satisfied in the phase model will be discussed. In Sec. IV, we introduce a specific vector oscillator model for globally coupled oscillators, and exhibit numerically that heteroclinic loops are formed in our vector model. We show there that the phase-coupling function, derived numerically from the vector model by the method of the phase reduction, satisfies the above-mentioned condition, leading to the formation of heteroclinic loops. In Sec. V, we generalize the argument in Sec. III to the vector model.

The formation of heteroclinic loops in globally coupled

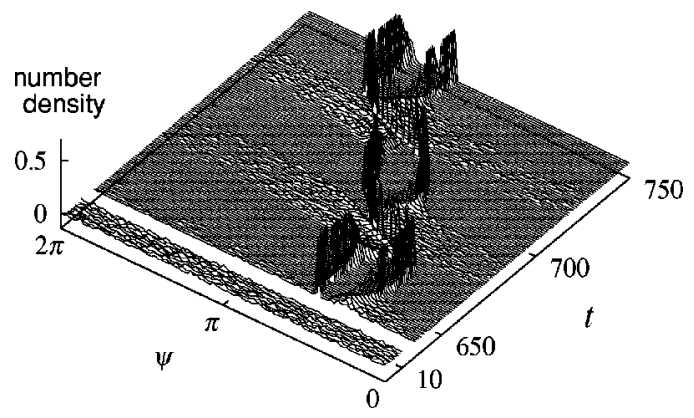

FIG. 1. Slow switching exhibited by the model in Ref. [10]. The figure displays the time evolution of the number density of the oscillators as a function of the phase. The whole population, which was initially almost uniform, splits into two subpopulations, each almost converging to a point cluster. After some time, however, this seeming convergence turns out to be unstable, and is followed by a period of scattering, but this again is followed by a period of convergence, and so forth. This form of alternation between the two characteristic periods of the convergence and dispersion of the clusters is called slow switching. The phase-advanced and phase retarded cluster at the end of one cycle becomes phase retarded and phase advanced at the end of the next cycle. 
oscillators may seem to be a pathological phenomenon which can occur only exceptionally. However, the time delay in coupling can easily cause a bifurcation from perfect synchrony to the formation of a heteroclinic loop, and this will be discussed in Sec. VI. The method of phase reduction provides a clear understanding of why this is actually possible. Slow switching becomes persistent when oscillators are subject to weak external noise, which will be discussed in Sec. VII by using a vector model. There we will also show that the same phenomenon can also be caused by other types of randomness.

\section{HETEROCLINIC LOOP IN THE PHASE MODEL}

Populations of weakly coupled limit cycle oscillators can be described by the phase model [5]. Suppose that the oscillators are identical, each interacting with all the others with equal strength. Then the corresponding phase model is expressed as

$$
\frac{d}{d t} \psi_{i}(t)=\omega+\frac{K}{N} \sum_{j=1}^{N} \Gamma\left[\psi_{i}(t)-\psi_{j}(t)\right]
$$

where $\psi_{i}(t) \quad\left(0 \leqslant \psi_{i}<2 \pi\right)$ is the phase of the $i$ th oscillator $(i=1, \ldots, N), \omega$ and $K$ are positive constants, and $\Gamma(x)$ is a coupling function with $2 \pi$ periodicity.

Hansel et al. [10] analyzed the case of a particular form of the coupling function

$$
\Gamma(x)=-\sin (x+1.25)+0.25 \sin (2 x) .
$$

They showed by numerical simulations that oscillators with random initial distributions are assembled to form two subgroups each in perfect phase synchrony, but with a constant mutual phase difference. The collective behavior of the system can conveniently be described in terms of the order parameter defined by

$$
O(t)=\frac{1}{N}\left|\sum_{j=1}^{N} \exp \left[i \psi_{j}\right]\right| .
$$

Its value is 1 for perfect synchrony, and 0 for perfect incoherence. A time trace of the order parameter for the above model is displayed in Fig. 2. Oscillators belonging to the respective groups are identical in phase, and this pair of point clusters rotates rigidly at a constant angular frequency. The mutual phase difference is denoted by $\Delta$. Hereafter we call the phase-advanced and retarded clusters $A$ and $B$ clusters, respectively. Let the fraction of the oscillators belonging to the $A$ cluster be $p$. Such a two-cluster state may thus be specified by $(p, \Delta)$, where $\Delta$ is within the region $-\pi<\Delta$ $\leqslant \pi$ by convention. This set of values may generally differ for different initial conditions.

The existence and stability of the two-cluster states can be analyzed as follows. Consider a two-cluster state with phases $\psi_{A}$ and $\psi_{B}$. Equation (1) then becomes

$$
\frac{d}{d t} \psi_{A}(t)=\omega+K\{p \Gamma(0)+(1-p) \Gamma(x)\},
$$

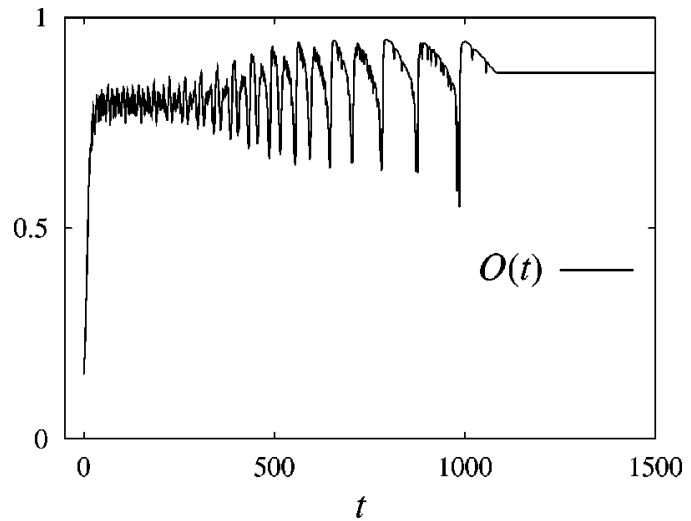

FIG. 2. Long transient of the order parameter after which the whole population converges to a two-cluster state.

$$
\frac{d}{d t} \psi_{B}(t)=\omega+K\{(1-p) \Gamma(0)+p \Gamma(-x)\},
$$

where $x$ denotes the phase difference, i.e., $x \equiv \psi_{A}-\psi_{B}$. Subtracting Eq. (5) from Eq. (4), we obtain

$$
\frac{d}{d t} x(t)=K\{(2 p-1) \Gamma(0)+(1-p) \Gamma(x)-p \Gamma(-x)\} .
$$

Since $x$ is constant in the two-cluster state, we have

$$
p(\Delta)=\frac{\Gamma(0)-\Gamma(\Delta)}{2 \Gamma(0)-\Gamma(\Delta)-\Gamma(-\Delta)} .
$$

$(p, \Delta)$ exists with $p$ satisfying $0<p<1$. Substituting Eq. (2) into Eq. (7), we obtain the condition for the existence of $(p, \Delta)$, and this is displayed graphically in Fig. 3. $\Delta$ takes three values for a given $p$ within the range $p_{\min }<p<1$ $-p_{\min }$, where $p_{\min }$ is defined by the minimum value of $p$ in the range $0<\Delta<\pi$. These three states are denoted by $\left(p, \Delta^{\prime}\right),\left(p,-\Delta^{\prime \prime}\right)=\left(1-p, \Delta^{\prime \prime}\right)$, and $\left(p, \Delta^{\prime \prime \prime}\right)$, where $\Delta^{\prime}$ and $\Delta^{\prime \prime}$ are understood to be positive and $\left|\Delta^{\prime \prime \prime}\right|$ to be larger than $\Delta^{\prime}$ and $\Delta^{\prime \prime}$.

The eigenvalues of the stability matrix are given by

$$
\lambda_{0}=0,
$$

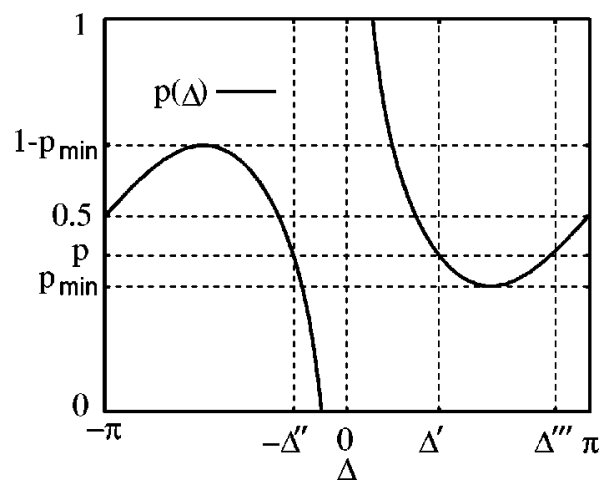

FIG. 3. Condition for the existence of two-cluster states. $\Delta$ takes three values for a given $p$ within the range $p_{\min }<p<1-p_{\min }$. 


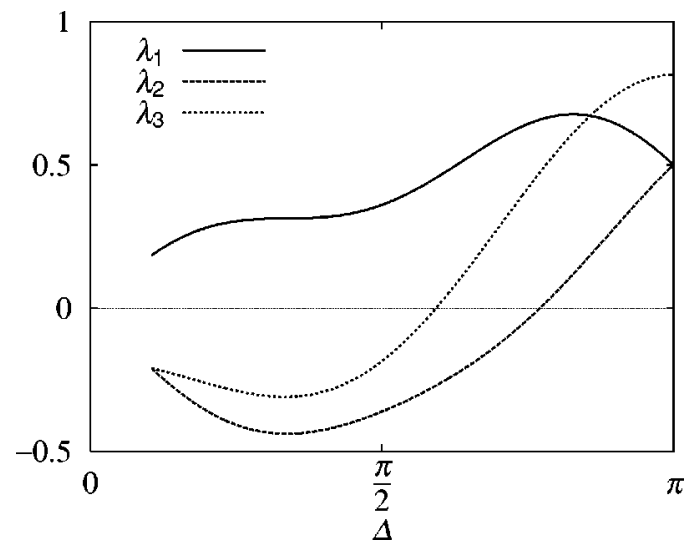

FIG. 4. Eigenvalues about two-cluster states as a function of $\Delta$. All two-cluster states are unstable here. Note that the eigenvalues $\lambda_{2}$ and $\lambda_{3}$ are negative for the states $\left(p, \Delta^{\prime}\right)$ and $\left(1-p, \Delta^{\prime \prime}\right)$.

$$
\begin{gathered}
\lambda_{1}=K\left\{p \Gamma^{\prime}(0)+(1-p) \Gamma^{\prime}(\Delta)\right\}, \\
\lambda_{2}=K\left\{(1-p) \Gamma^{\prime}(0)+p \Gamma^{\prime}(-\Delta)\right\}, \\
\lambda_{3}=K\left\{(1-p) \Gamma^{\prime}(\Delta)+p \Gamma^{\prime}(-\Delta)\right\},
\end{gathered}
$$

with multiplicities $1, N p-1, N(1-p)-1$, and 1 , respectively. $\Gamma^{\prime}(x)$ is defined as $(d / d x) \Gamma(x) . \lambda_{0}$, which vanishes identically, always exists due to the invariance of Eq. (4) with respect to a constant shift of $\psi_{A}$ and $\psi_{B} . \lambda_{1}$ and $\lambda_{2}$ are associated with the fluctuations of the individual oscillators belonging to the $A$ cluster and the $B$ cluster, respectively. $\lambda_{3}$ corresponds to the fluctuation in $\Delta$. Figure 4 displays the eigenvalues versus $\Delta$ obtained using Eq. (2) with $K=1$, which shows that all two-cluster states are unstable. It is important to note that $\left(p, \Delta^{\prime}\right)$ and $\left(1-p, \Delta^{\prime \prime}\right)$ are saddles which have negative eigenvalues of $\lambda_{2}$ and $\lambda_{3}$. $\left(p, \Delta^{\prime \prime \prime}\right)$, however, has a positive $\lambda_{3}$, which can be verified by the property $\lambda_{3} \propto(d / d \Delta) p(\Delta)$.

Paradoxically, the system converges to unstable solutions. This counterintuitive fact may be understood if we assume that the pair of saddles $\left(p, \Delta^{\prime}\right)$ and $\left(1-p, \Delta^{\prime \prime}\right)$ are connected heteroclinically $[4,10]$. All numerical results in Ref. [10] support this assumption. Although the heteroclinicity is considered structurally unstable, this does not seem to apply to the particular class of systems under consideration. In Sec. III it will be confirmed that $\left(p, \Delta^{\prime}\right)$ and $\left(1-p, \Delta^{\prime \prime}\right)$ are in fact connected heteroclinically through an invariant subspaces, and it will be argued how this structure is stably maintained.

\section{STRUCTURE OF THE HETEROCLINIC LOOP}

We first note a particular symmetry of our phase model given by Eq. (1), which is expressed as

$$
\left.\frac{d}{d t}\left\{\psi_{i}(t)-\psi_{j}(t)\right\}\right|_{\psi_{i}(t)=\psi_{j}(t)}=0 \quad \text { for all } i, j .
$$

The above equation shows that, when the phases of some oscillators are found to be identical at some time, they will

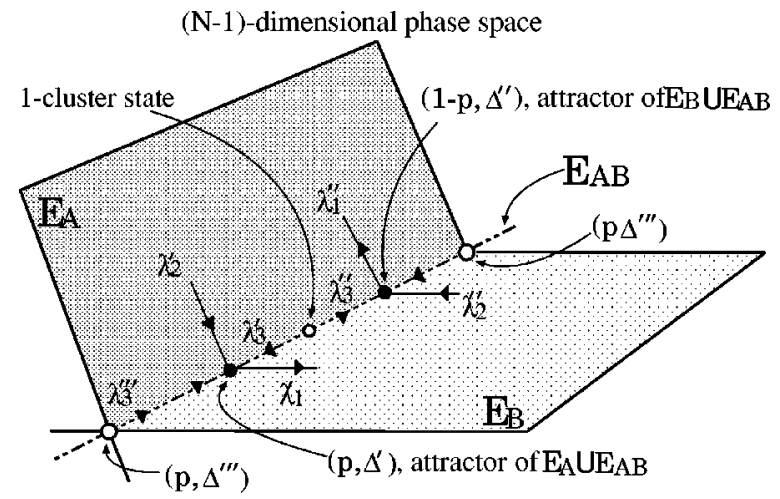

FIG. 5. Schematic representation of the structure of a heteroclinic loop. $\left(p, \Delta^{\prime}\right)$ and $\left(1-p, \Delta^{\prime \prime}\right)$ are the attractors of the invariant subspace $E_{A} \cup E_{A B}$ and $E_{B} \cup E_{A B}$, respectively.

obey completely the same dynamics thereafter, namely, a point cluster remains a point cluster forever. This property will turn out to be crucial to the formation of heteroclinic loops.

We now argue how phase model (1) can form heteroclinic loops which are structurally stable. A generalization to the vector model of the limit cycle oscillators will be given in the subsequent sections. The existence of a heteroclinic loop connecting $\left(p, \Delta^{\prime}\right)$ and $\left(1-p, \Delta^{\prime \prime}\right)$ is clear if the following properties are satisfied:

$(\mathrm{X})\left(p, \Delta^{\prime}\right)$ is a global attractor of $W^{\mathrm{u}}\left(1-p, \Delta^{\prime \prime}\right)$,

$(\mathrm{Y})\left(1-p, \Delta^{\prime \prime}\right)$ is a global attractor of $W^{\mathrm{u}}\left(p, \Delta^{\prime}\right)$,

where $W^{\mathrm{u}}(p, \Delta)$ represents the unstable manifold of $(p, \Delta)$. We work with an $(N-1)$-dimensional phase space throughout, by which the degree of freedom associated with a rigid rotation of the entire system is ignored. For an aid to the understanding of a slightly complicated situation, it would be appropriate to display in advance a schematic picture of the heteroclinic loop under consideration in Fig. 5, where $\lambda_{i}^{\prime}$, $\lambda_{i}^{\prime \prime}$, and $\lambda_{i}^{\prime \prime \prime}(i=1,2$, and 3$)$ are the eigenvalues of $\left(p, \Delta^{\prime}\right)$, $\left(1-p, \Delta^{\prime \prime}\right)$, and $\left(p, \Delta^{\prime \prime \prime}\right)$, respectively. The definition of $E_{X}$ ( $X=A, B$, and $A B$ ) will be given later.

Our argument will be based on the assumptions that there are three two-cluster states in the range $p_{\min }<p<1-p_{\min }$ and that the eigenvalues associated with these solutions satisfy certain stability properties. Specifically, the assumptions may be summarized as follows:

(a) $\left(p, \Delta^{\prime}\right),\left(1-p, \Delta^{\prime \prime}\right)$, and $\left(p, \Delta^{\prime \prime \prime}\right)$ exist,

(b) $\lambda_{1}^{\prime}>0$,

(c) $\lambda_{2}^{\prime}<0$ and $\lambda_{3}^{\prime}<0$,

(d) $\lambda_{1}^{\prime \prime}>0$,

(e) $\lambda_{2}^{\prime \prime}<0$ and $\lambda_{3}^{\prime \prime}<0$,

(f) $\lambda_{3}^{\prime \prime \prime}>0$, 
(g) $\lambda_{1}$ of all two-cluster states are positive,

(h) $\Gamma^{\prime}(x=0)>0$.

Here we assumed that both $N p$ and $N(1-p)$ are larger than 1 , so that three independent eigenvalues $\lambda_{i}(i=1,2$, and 3) exist. Note that all the assumptions are satisfied by Eq. (1) with the coupling function given by Eq. (2), as stated in Sec. II.

Consider the tangent space around $\left(p, \Delta^{\prime}\right) .\left(p, \Delta^{\prime}\right)$ has $N p-1$ degenerate eigenvalues given by $\lambda_{1}^{\prime}$. Thus the corresponding eigenvectors span an $(N p-1)$-dimensional unstable eigenspace which is denoted by $E_{B}$. Similarly, the eigenvectors corresponding to $\lambda_{2}^{\prime}$ span an $[N(1-p)-1]$-dimensional stable eigenspace which is denoted by $E_{A}$. An eigenvector corresponding to $\lambda_{3}$ spans the one-dimensional stable eigenspace $E_{A B}$. In particular, the following statements hold:

(b') $E_{B}$ is an unstable subspace of $\left(p, \Delta^{\prime}\right)$.

$\lambda_{1}^{\prime}$ corresponds to the fluctuations which occur in the $A$ cluster (i.e., the phase-advanced cluster). Thus the eigenspace $E_{B}$ is associated with the disintegration of the $A$ cluster, while the $B$ cluster remains a point cluster. Similarly, the $B$ cluster is disintegrated in the eigenspace $E_{A}$, while the $A$ cluster remains a point cluster there. In the space $E_{A B}$, in contrast, these clusters remain point clusters while their mutual distance changes. Since a point cluster must remain a point cluster at any time, as noted at the beginning of this section, the space $E_{B} \cup E_{A B}$, on which the $B$ cluster is a point cluster, gives an invariant subspace of dimension $N p$. Similarly, $E_{A} \cup E_{A B}$, on which the $A$ cluster is a point cluster, is an invariant subspace of dimension $N(1-p)$. Note that the unstable manifold $W^{\mathrm{u}}\left(p, \Delta^{\prime}\right)$ must coincide with $E_{B}$ in the vicinity of $\left(p, \Delta^{\prime}\right)$. This fact, combined with the obvious fact that $E_{B}$ is included in the invariant subspace $E_{B} \cup E_{A B}$, leads to the following statements which hold globally.

(x1) $W^{\mathrm{u}}\left(p, \Delta^{\prime}\right)$ is included by the invariant subspace

$$
E_{B} \cup E_{A B} \text {. }
$$

Arguments parallel to the above can be developed around $\left(1-p, \Delta^{\prime \prime}\right)$, i.e., the state where the $B$ cluster is phase advanced by $\Delta^{\prime \prime}$. From the assumed property (e), $E_{B} \cup E_{A B}$ is the stable subspace of $\left(1-p, \Delta^{\prime \prime}\right)$, which can be restated as

$\left(\mathrm{e}^{\prime}\right)\left(1-p, \Delta^{\prime \prime}\right)$ is an attractor of $E_{B} \cup E_{A B}$.

Therefore, if

(x2) $\left(1-p, \Delta^{\prime \prime}\right)$ is a unique attractor of $E_{B} \cup E_{A B}$,

then we may assume

(x3) $\left(1-p, \Delta^{\prime \prime}\right)$ is a global attractor of $E_{B} \cup E_{A B}$. (x1) and (x3) give the sufficient conditions for (X) to hold. A similar discussion can be developed to derive $(\mathrm{Y})$ via the assumption

(y2) $\left(p, \Delta^{\prime}\right)$ is a unique attractor of $E_{A} \cup E_{A B}$.

Note that $\left(p, \Delta^{\prime \prime \prime}\right)$ gives a source point lying between $\left(p, \Delta^{\prime}\right)$ and $\left(1-p, \Delta^{\prime \prime}\right)$ in the phase space, as displayed in Fig. 5.

There still remains a problem about the validity of assumptions (x2) and (y2). It is sufficient to consider assumption (x2) only. If the type of attractor is limited to the twocluster state, then it is obvious that $\left(1-p, \Delta^{\prime \prime}\right)$ is a unique attractor of $E_{B} \cup E_{A B}$, as can be confirmed by property $(\mathrm{g})$. How about the possibility for a one-cluster state, namely, perfect synchrony, to become an attractor? The eigenvalues of the one-cluster state are $(N-1)$-fold degenerate, and given by $K \Gamma^{\prime}(x=0)$, which is positive by the assumption (h) so that there is no stable manifold of one-cluster state. How about the stability structure of $n(\geqslant 3)$-cluster states? They could be attractors of the invariant subspaces for the same reason as the two-cluster states, even if they are unstable solutions. In the case of three or more clusters, however, the resulting heteroclinicity would be even more complicated. Numerical simulations in Sec. II, however, displayed the simple heteroclinicity between pairs of twocluster solutions, implying the validity of the assumption (x2) and (y2) in Eq. (1) with Eq. (2).

Convergence to the heteroclinic loop can be discussed similarly to the case of a heteroclinic orbit in a twodimensional phase space, which was discussed in Ref. [10]. The result is that the system which is initially close to a heteroclinic loop converges to it provided

$$
\frac{\lambda_{1}^{\prime} \lambda_{1}^{\prime \prime}}{\lambda_{2}^{\prime} \lambda_{2}^{\prime \prime}}<1 .
$$

If this condition is satisfied, the heteroclinic loop is attracting. In numerical simulations, this convergence is established in a finite time due to the roundoff errors.

Although we have assumed conditions (a)-(h) so far, our discussion is expected not to rely so heavily on the specific form of $\Gamma(x)$. In fact, these conditions may be satisfied for a broader class of the coupling function. For instance, they are satisfied if we assume a simple shape of the coupling function such that $\Gamma(x)$ decreases in the range $-\pi<x<0$, while it increases otherwise (see Fig. 6). The reason is the following. The corresponding shape of $p(\Delta)$ turns out similar to the curve in Fig. 3, so that we can define $p_{\min }$ similarly. For a given $p$ satisfying $p_{\min }<p<1-p_{\min }$, we obtain three states $\left(p, \Delta^{\prime}\right),\left(p,-\Delta^{\prime \prime}\right)=\left(1-p, \Delta^{\prime \prime}\right)$, and $\left(p, \Delta^{\prime \prime \prime}\right) . \lambda_{1}$ of each $(p, \Delta>0)$ is positive because $\Gamma^{\prime}(0 \leqslant x \leqslant \pi)>0$. We can verify $\lambda_{3}^{\prime}<0, \lambda_{3}^{\prime \prime}<0$ and $\lambda_{3}^{\prime \prime \prime}>0$ through the property that $\lambda_{3}$ is proportional to $(d / d \Delta) p(\Delta)$. The one-cluster state turns out to be unstable since $\Gamma^{\prime}(0)>0$. Hence we have confirmed assumptions (a)-(h) except for $\lambda_{2}^{\prime}, \lambda_{2}^{\prime \prime}<0$. For the last properties to be satisfied, we need one more assumption, that is, $\Gamma^{\prime}(0)$ is not so large as to admit a region of $p$ where both $\lambda_{2}^{\prime}$ and $\lambda_{2}^{\prime \prime}$ are negative. Such a region is expressed by $p^{*}$ $<p<1-p^{*}$, where $p^{*}$ satisfies $p_{\min } \leqslant p^{*}<0.5$. Then, via 


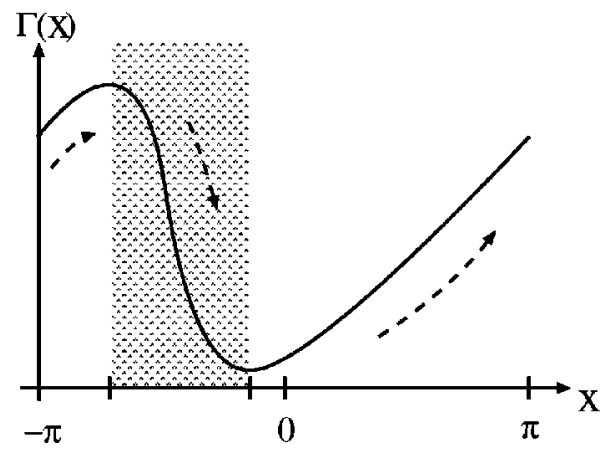

FIG. 6. Typical shape of the coupling function which admits heteroclinic loops. This shape lead to conditions (a)-(h) except for $\lambda_{2}^{\prime}, \lambda_{2}^{\prime \prime}<0$. The last property also holds if $\Gamma^{\prime}(0)$ is not too large.

assumptions (x2) and (y2), we obtain sufficient conditions for the existence of a heteroclinic loop between $\left(p, \Delta^{\prime}\right)$ and $\left(1-p, \Delta^{\prime \prime}\right)$ within the range $p^{*}<p<1-p^{*}$.

In previous works $[4,10]$, the shape of the employed coupling functions satisfied this property. Our model, discussed in Sec. IV, also fulfills this condition in the weak-coupling limit where the phase description is valid. Thus we may regard the coupling function with this property of the shape as a typical class which admits heteroclinic loops.

The existence of a phase space structure yielding a heteroclinic loop has thus been confirmed, which can be summarized as follows. For a given coupling function $\Gamma(x)$, we can easily verify whether conditions (a)-(h) are satisfied. Among these conditions, (a)-(f) constitute a necessary condition for the existence of a heteroclinic loop between $\left(p, \Delta^{\prime}\right)$ and $\left(1-p, \Delta^{\prime \prime}\right)$, while conditions $(\mathrm{g})$ and (h) support assumptions (x2) and (y2). One may also consider the case where the roles of $\lambda_{1}$ and $\lambda_{2}$ are reversed. The saddle connections are stably formed through the invariant subspace which exists for the symmetry of equations of motion, or Eq. (12). Thus we conclude that the heteroclinic loop is robust under such small perturbations that maintain the homogeneity of the population and the symmetry of the global coupling.

\section{COUPLED LIMIT CYCLE OSCILLATORS}

Our argument on the existence and structural stability of the heteroclinic loop developed in Sec. III was based on the phase model, with some assumed properties of the phasecoupling function. In this section, we discuss a specific coupled oscillator model in which heteroclinic loops are formed. From the model, a phase-coupling function of the desired properties for the existence of heteroclinic loops is derived through the method of phase reduction. To our knowledge, the existence of a heteroclinic loop associated with slow switching has never been reported for vector models of oscillators.

Consider a general system of coupled oscillators which are identical and all to all coupled:

$$
\frac{d}{d t} \boldsymbol{X}_{i}(t)=\boldsymbol{F}\left(\boldsymbol{X}_{i}\right)+\frac{K}{N} \sum_{j=1}^{N} \boldsymbol{G}\left(\boldsymbol{X}_{i}, \boldsymbol{X}_{j}\right) .
$$

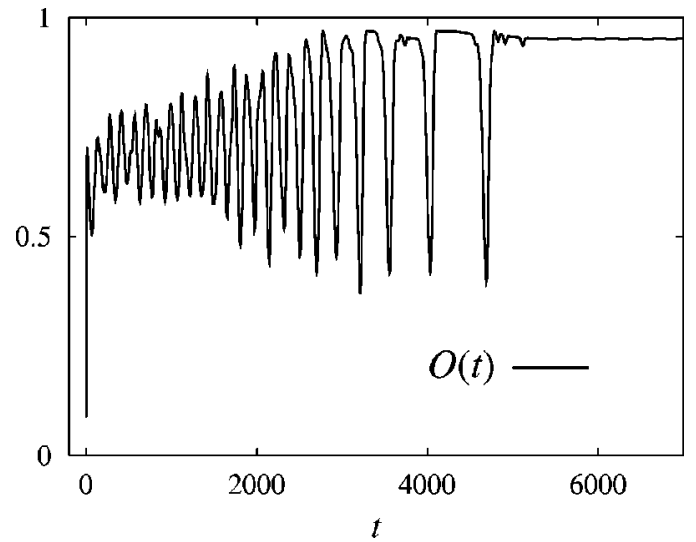

FIG. 7. Time series of the order parameter. The order parameter $O$ is conveniently defined in the following way. Let $t_{j}$ $(j=0,1,2, \ldots)$ denote the time at which the representative point of the $j$ th oscillator crosses a given section $\Sigma$ in the one-oscillator phase space. The order parameter at time $t=t_{N}$ is defined as

$$
O\left(t=t_{N}\right)=\frac{1}{N}\left|\sum_{j=1}^{N} \exp \left[i \frac{2 \pi\left(t_{j}-t_{0}\right)}{t_{N}-t_{0}}\right]\right|
$$

as a generalization of Eq. (3). Since the oscillators cross $\Sigma$ again and again, the order parameter at discrete times $t=t_{k N}(k$ $=1,2, \ldots$ ) may be defined similarly. Note that $O(t)=1$ when the oscillators are synchronized perfectly, and $O(t)=0$ when their phases are uniformly distributed.

Here $\boldsymbol{X}_{i}, \boldsymbol{F}$, and $\boldsymbol{G}$ are $m$-dimensional real vectors, and $K$ is a positive constant. Note that Eq. (14) satisfies the condition

$$
\left.\frac{d}{d t}\left\{\boldsymbol{X}_{i}(t)-\boldsymbol{X}_{j}(t)\right\}\right|_{\boldsymbol{X}_{i}(t)=\boldsymbol{X}_{j}(t)}=0 \quad \text { for all } i, j,
$$

which is similar to Eq. (12). Suppose that the local dynamics is two dimensional, i.e., $\boldsymbol{X}=(x, y)$, and the specific forms of $\boldsymbol{F}$ and $\boldsymbol{G}$ are given by

$$
\begin{gathered}
\boldsymbol{F}\left(\boldsymbol{X}_{i}\right)=\left(\begin{array}{l}
F_{x} \\
F_{y}
\end{array}\right)=\left(\begin{array}{l}
3 x_{i}^{2}-x_{i}^{3}+y_{i}-\mu \\
1-5 x_{i}^{2}-y_{i}
\end{array}\right), \\
\boldsymbol{G}\left(\boldsymbol{X}_{i}, \boldsymbol{X}_{j}\right)=\left(\begin{array}{l}
G_{x} \\
G_{y}
\end{array}\right)=\left(\begin{array}{l}
x_{j}-x_{i} \\
0
\end{array}\right) .
\end{gathered}
$$

The corresponding equation $\dot{\boldsymbol{X}}=\boldsymbol{F}$ is called the HindmarshRose model [11], which was originally proposed for a neural oscillator. Without coupling, i.e. $K=0$, each unit becomes oscillatory if $-11.5<\mu<0.8$ [12]. The coupling is assumed to be diffusive, and in terms of neurophysiology this corresponds to the electrical synapse formed by gap junctions [13].

The parameter values are set to $K=0.1, N=100$, and $\mu$ $=-1$. The intrinsic frequency then becomes $\omega \simeq 1.0$. We choose random initial conditions. Some numerical results obtained are summarized as follows. The system converges after a long transient to a two-cluster state which is periodic in time. Figure 7 displays a time series of the order parameter. The relative population of the clusters generally depends on the initial condition. Convergence to the two-cluster state 


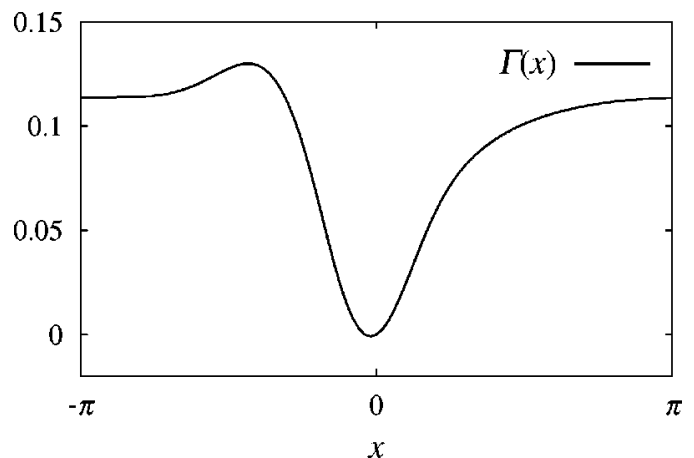

FIG. 8. Coupling function of the reduced model. The minimum of $\Gamma(x)$ appears at a negative $x$. Such a shape of $\Gamma$ is typical when heteroclinic loops exist.

does not imply its stability, and is rather due to numerical artifacts. Actually, when very small perturbations are given to the oscillators independently, the clusters start to disintegrate, implying their linear instability. Such a behavior is very similar to that of the phase model when heteroclinic loops exist. We now show that the phase reduction of the above model produces a phase-coupling function which admits, based on the argument of Sec. III, heteroclinic loops.

Coupled oscillators can be reduced to the phase model [Eq. (1)] when the coupling is sufficiently weak [5]. There is a general formula for the phase-coupling function, and for a given dynamical-system model, this can be computed numerically. We did this for Eqs. (14), (16) and (17). The coupling function $\Gamma(x)$ obtained is displayed in Fig. 8, which shows a typical shape admitting heteroclinic loops (see Fig. 6). Two-cluster solutions were sought, and their stability analysis was done through Eqs. (7)-(11). Then we confirmed that the reduced model satisfies conditions (a)-(h) for the existence of a heteroclinic loop and also condition (13) for its stability.

\section{STRUCTURE OF THE HETEROCLINIC LOOP IN VECTOR MODELS}

The preceding arguments clarify the nature of the heteroclinic loop in the framework of the phase model. In this section, we show that such arguments can be generalized to the original model for coupled limit-cycle oscillators in vector form.

It would be appropriate to start to reconsider the model given by Eqs. (14), (16) and (17). Under suitable initial conditions, we obtain various two-cluster states. They correspond to the solutions $(p, \Delta)$ with $\lambda_{3}<0$ in the phasecoupling limit, and these two-cluster states will be denoted by $p$ states. Also, clusters corresponding to the phaseadvanced and -retarded clusters will be called the $A$ and $B$ clusters, respectively. For a given $p$ state, $m N$ Lyapunov eigenvalues can be computed numerically, where $m=2$ for the model under consideration. They can be classified into four groups $\Lambda_{0}, \Lambda_{1} s, \Lambda_{2} s$ and $\Lambda_{3} s$, and they will degenerate respectively into $\lambda_{i}(i=0,1,2$, and 3$)$ in the phasecoupling limit. Note that each of $\Lambda_{1} s$ and $\Lambda_{2} s$ is composed of $m$ eigenvalues, while $\Lambda_{3} s$ is composed of $2 m-1$ eigen-

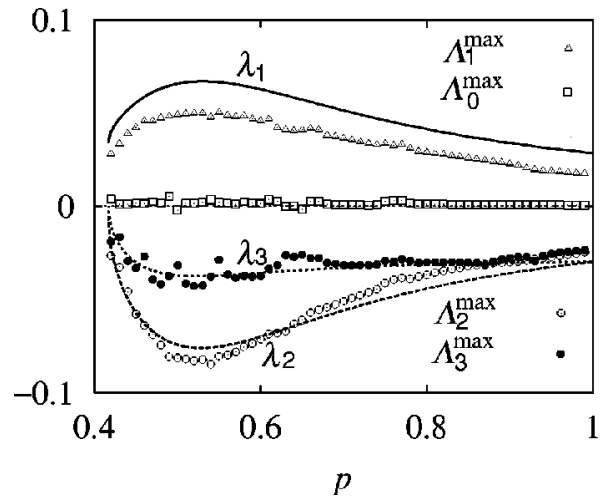

FIG. 9. Lyapunov spectrum plotted against $p$. From the figure, it can be seen that $p_{\text {min }} \simeq 0.42$. Solid lines show the eigenvalues obtained by the phase reduction, with excellent agreement with the spectrum of the original system.

values. $\Lambda_{1} s$ corresponds to fluctuations within the $A$ cluster, and each eigenvalue of this group is $(N p-1)$-fold degenerate. Similarly, $\Lambda_{2} s$ corresponds to fluctuations within the $B$ cluster, and each eigenvalue there is $[N(1-p)-1]$-fold degenerate. $\Lambda_{3} s$ is associated with the relative motion between the clusters. $\Lambda_{0}$ is identical to 0 , resulting from the time periodicity of the solutions. The maximum value of $\Lambda_{i} s$ ( $i$ $=1,2$, and 3) is denoted by $\Lambda_{i}^{\max }$, and their numerical values are displayed in Fig. 9.

The argument on the structure of a heteroclinic loop connecting the $p$ state and $(1-p)$ state can be developed quite similar to that in Sec. III. We now have to work with the $(m N-1)$-dimensional phase space, whereby we employ a surface of section to remove the irrelevant degrees of freedom corresponding to a steady rotation of the whole population. The eigenspaces $E_{A}$ and $E_{B}$, associated with $\Lambda_{1} s$ of the $p$ state and the $(1-p)$ state, are now $m\{(N(1-p)-1\}$ dimensional and $m(N p-1)$ dimensional, respectively, while the eigenspace $E_{A B}$ associated with $\Lambda_{3} s$ is $(2 m-1)$ dimensional. Then the argument in Sec. III still holds if we replace $\lambda_{i}$ with $\Lambda_{i}^{\max }$. Note that we assume the existence of an unstable state corresponding to $\left(p, \Delta^{\prime \prime \prime}\right)$ which is hard to obtain numerically.

The eigenvalues $\Lambda_{i}^{\max }$ are the ones which should coincide with $\lambda_{i}$ in the phase-oscillator limit. As the coupling becomes stronger, the heteroclinic loop can persist as far as the existence and stability properties of the two point clusters are unchanged. Generally speaking, stronger coupling makes point clusters more stable. In Fig. 9, this effect is already sizable for $K=0.1$. As $K$ becomes $O(1)$, the two-cluster state gives way to a one-cluster state by which the heteroclinic loop disappears. We expect in general that the heteroclinic loop disappears when the coupling is so strong that the phase description completely breaks down.

\section{APPEARANCE OF HETEROCLINIC LOOPS THROUGH DELAY-INDUCED BIFURCATIONS}

In globally coupled identical oscillators, a one-cluster state is the easiest state to appear. This can be illustrated by 
the following form of coupling:

$$
\boldsymbol{G}\left(\boldsymbol{X}_{i}, \boldsymbol{X}_{j}\right)=\boldsymbol{X}_{j}(t)-\boldsymbol{X}_{i}(t)
$$

Assuming Eqs. (14), (16), and (18), we obtain a stable onecluster state even if $K$ is very small. We may generally expect that heteroclinic loops cannot exist when the one-cluster state is stable. In the above model, it can be shown that time-delayed coupling causes an instability of the one-cluster state, which at the same time is accompanied by the appearance of the heteroclinic loop. The corresponding bifurcation is a so-called transcritical bifurcation.

Consider uniformly delayed coupling

$$
\boldsymbol{G}\left(\boldsymbol{X}_{i}, \boldsymbol{X}_{j}\right)=\boldsymbol{X}_{j}(t-\tau)-\boldsymbol{X}_{i}(t)
$$

where $\tau$ denotes the delay. Note that the symmetry property [Eq. (15)] still holds when the coupling involves a uniform delay of the form of Eq. (19). We will show some numerical results obtained for the system given by Eqs. (14), (16), and (19), where the parameter values are the same as in Sec. IV. Without delay, the system under various initial conditions immediately converges to a one-cluster state. As $\tau$ is increased, the one-cluster state persists up to a critical value $\tau_{c}$, beyond which the cluster splits in two and at the same time heteroclinic loops are formed. In this case of the parameters, this critical value is about 0.18 .

This result can be understood by a phase reduction of our model which is applicable when the coupling is weak. The reduced model takes the form

$$
\frac{d}{d t} \psi_{i}(t)=\omega+\frac{K}{N} \sum_{j=1}^{N} \Gamma\left[\psi_{i}(t)-\psi_{j}(t-\tau)\right],
$$

where $\omega \simeq 1.0$ at $\mu=-1$. Since the second term on the righthand side is much smaller than the first term by assumption, Eq. (20) is further reduced to the form

$$
\frac{d}{d t} \psi_{i}(t)=\omega+\frac{K}{N} \sum_{j=1}^{N} \Gamma\left[\psi_{i}(t)-\psi_{j}(t)+\omega \tau\right] .
$$

Thus there is no explicit delay in coupling, while its effect has now been converted to a phase shift of the coupling function by $\omega \tau$. The situation is illustrated in Fig. 10. The stability of a one-cluster state depends entirely on the sign of $\Gamma^{\prime}(\omega \tau)$. Thus the one-cluster state is stable for small $\tau$, which admits $\Gamma^{\prime}(\omega \tau)<0$. As $\tau$ is increased, the one-cluster state becomes less stable, and, at $\tau=x_{0} / \omega$, it becomes unstable where $x_{0}$ is defined as the value of $x$ which minimizes $\Gamma(x)$. Note that $\tau_{c}$, obtained numerically, would agree with $x_{0} / \omega(\simeq 0.13)$ for sufficiently small $K$. For $\tau>x_{0} / \omega$, the coupling function assumes a typical shape under which a heteroclinic loop exists. At $\tau=0.3$, for example, conditions (a)-(h) and (13) are satisfied in this reduced model. This transition occurs through a transcritical bifurcation.

Let $x$ denote the mutual phase difference between the clusters. The equation obeyed by $x$ can be derived similarly to the derivation of Eq. (6), and takes the form

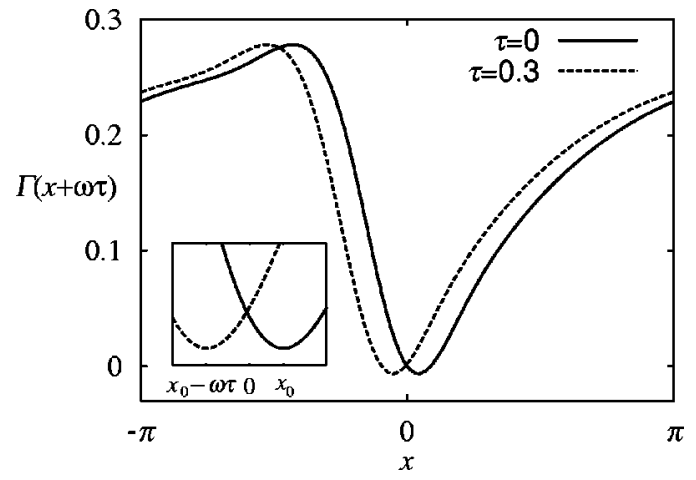

FIG. 10. Coupling functions. The solid line is obtained from Eqs. (14), (16), and (19) with $\tau=0$, while the dotted line is obtained just by a phase shift of solid line by $-\omega \tau$. The effect of the delay is equivalent to a simple modification of the coupling function in the weak-coupling limit. The modified coupling function is a typical shape admitting heteroclinic loops, provided the condition $\tau>x_{0} / \omega$ is satisfied.

$$
\begin{aligned}
\frac{d}{d t} x(t)= & K\{(2 p-1) \Gamma(\omega \tau)+(1-p) \Gamma(x+\omega \tau) \\
& -p \Gamma(-x+\omega \tau)\}
\end{aligned}
$$

The above equation has a trivial solution $x=0$. For small $x$, the right-hand side can be expanded in powers of $x$. We find that, provided $p \neq 0.5$ the right-hand side involves an $x^{2}$ term as the lowest nonlinearity. This means that the trivial solution loses stability via a transcritical bifurcation. This occurs at $\tau=x_{0} / \omega$. We also find that as $\tau$ is increased a saddle-node bifurcation occurs slightly before the transcritical bifurcation. For $\tau>x_{0} / \omega$, the stable manifolds associated with the saddle-node and transcritical bifurcations connect smoothly and form a heteroclinic loop. Let $y(z)$ denote a certain direction taken on $E_{B}\left(E_{A}\right)$. How the bifurcation in question occurs is explained schematically in Fig. 11. The same structure of bifurcation leading to the formation of heteroclinic loops holds for some range of $p$ where $\lambda_{2}^{\prime}$ and $\lambda_{2}^{\prime \prime}$ are both negative. Note that at $p=0.5$ the term of $x^{2}$ vanishes so that a pitchfork bifurcation occurs at $\tau=x_{0} / \omega$ and no saddle-

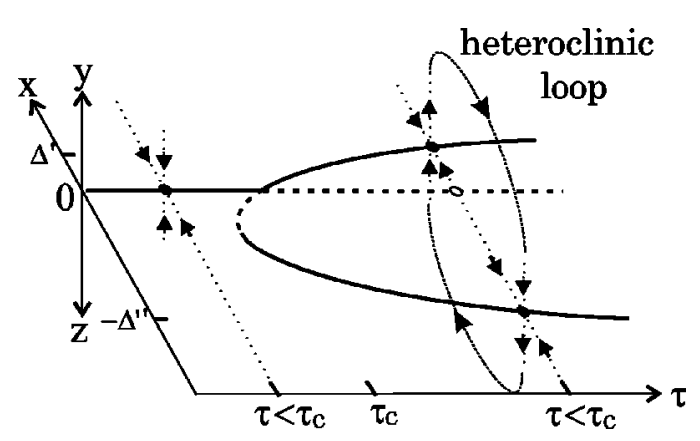

FIG. 11. Schematic representation of the bifurcation structure. The trivial solution $x=0$ loses its stability at $\tau=\tau_{c}$ via a transcritical bifurcation. Two solid lines existing for $\tau>\tau_{\mathrm{c}}$ correspond to $\left(p, \Delta^{\prime}\right)$ and $\left(1-p, \Delta^{\prime \prime}\right)$, respectively, each being unstable with respect to the $y$ or $z$ direction alternately. A heteroclinic loop is formed between these two solutions, as explained in Sec. III. 
node bifurcation occurs before. A heteroclinic loop is formed similarly to the case of $p \neq 0.5$.

In coupled oscillators, the appearance of the heteroclinic loop may seem to be pathological. The results in this section, however, imply that the heteroclinic loop appears in a broad class of weakly coupled oscillators, including those studied so far, provided a uniformly delayed coupling is introduced. Assuming a simple coupling such as Eq. (18), we find that when the assemblies are composed of relaxation oscillators, their phase coupling function is often characterized by a curve which sharply decreases in a small region while it gradually increases otherwise. Thus heteroclinic loops are expected to arise in homogeneous assemblies of relaxation oscillators.

\section{SLOW SWITCHING}

When the system involves heteroclinic loops, it exhibits a remarkable dynamics when perturbed weakly. In the analysis of a model of the form of Eq. (1), Hansel et al. [10] applied week noise independently to individual oscillators, and observed the appearance of a very long time scale depending on the noise intensity (see Fig. 1). Since the time scale here is associated with an alternation between two collective states (i.e., a pair of two-cluster states), they called this characteristic behavior of the system slow switching, and gave a successful explanation for it in terms of a weakly perturbed heteroclinic loop. Their explanation may be summarized as follows. If a heteroclinic loop is attracting, i.e., if Eq. (13) is satisfied, then the system approaches one saddle and then other alternately. Without noise, the minimal distance from each saddle should decrease exponentially in time. With noise, however, this distance will fluctuate but remain finite typically within the order $\sigma$, the square root of the variance of the noise. In any case, the system for the most part stays close to one saddle or the other, so that the dynamics could be characterized dominantly by the local properties around the saddles. The time interval $T$ for a stay near a saddle may be estimated as

$$
T \sim-\frac{1}{\lambda_{u}} \ln \sigma
$$

where $\lambda_{u}$ is the eigenvalue of the most unstable direction. Thus the period of the switching is logarithmically dependent on the noise intensity.

By including noise, Eq. (14) is generalized as

$$
\frac{d}{d t} \boldsymbol{X}_{i}(t)=\boldsymbol{F}\left(\boldsymbol{X}_{i}\right)+K \sum_{j=1}^{N} \boldsymbol{G}\left(\boldsymbol{X}_{i}, \boldsymbol{X}_{j}\right)+\sigma \boldsymbol{\xi}_{i}(t),
$$

where $\boldsymbol{\xi}_{i}$ is Gaussian white noise with variance 1 and the parameter $\sigma$, assumed to be small below, indicates the intensity of noise. Let us now consider specific forms for $\boldsymbol{F}$ and $\boldsymbol{G}$ given by Eqs. (16) and (19), respectively, where the parameter values are the same as before. Some results obtained numerically are the following. For $0 \leqslant \tau<\tau_{\mathrm{c}}$, the oscillators localize in a small phase range, which we call a noisy onecluster state. Figure 12 displays a time trace of the order

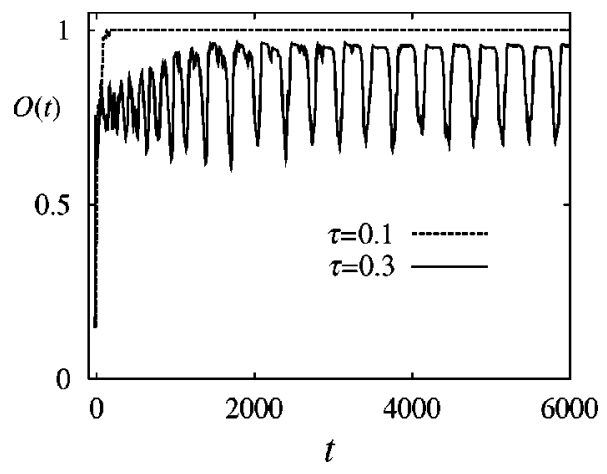

FIG. 12. Time series of the order parameter for a noisy system. The solid line shows slow switching, where a new time scale of dynamics appears.

parameter $O$, where $\tau=0.1$ and $\rho=10^{-7}$. It is seen that $O$ stays near 1 . For $\tau>\tau_{\mathrm{c}}$, this highly coherent cluster becomes unstable, and $O$ begins to oscillate. After a long transient, the system comes to exhibit a slow switching between a pair of two-cluster states. For the most part the system stays close to one of the noisy two-cluster states, which is followed by a short period of cluster disintegration, then by convergence to another two-cluster state. This is demonstrated in Fig. 14 for $\tau=0.3$. It is clear that the collective dynamics is then characterized by a new time scale corresponding to this slow switching. We define the period of switching $T$ as the average time between the two successive local minima of $O$ sufficiently after the transient. The logarithmic dependence of $T$ on the noise intensity is clear from Fig. 13. The steepness of the $T$ versus the $\ln \sigma$ curve after linear fitting is estimated as $|T / \ln \sigma| \simeq 20$, which suggests that $\Lambda_{1}^{\max }$ of this delayed coupling model is about $0.05 . \Lambda_{1}^{\max }$ can be easily estimated by Eq. (9) with the phase-shifted coupling function displayed in Fig. 10. We obtain $\lambda_{1} \simeq 0.065$ at $p=0.5$, which is close to the above estimation of $\Lambda_{1}^{\max }$ $\simeq 0.05$. Note that the amplitude effect makes $\Lambda_{1}^{\max }$ smaller than $\lambda_{1}$ similarly to the case of Fig. 9 .

Slow switching is thus the fate of the system when the heteroclinic loop is perturbed weakly. Besides external noise, a slight violation of the symmetry property [Eq. (15)] is expected to cause the same effect. Imagine a particular case where a heteroclinic loop is present under symmetry condi-

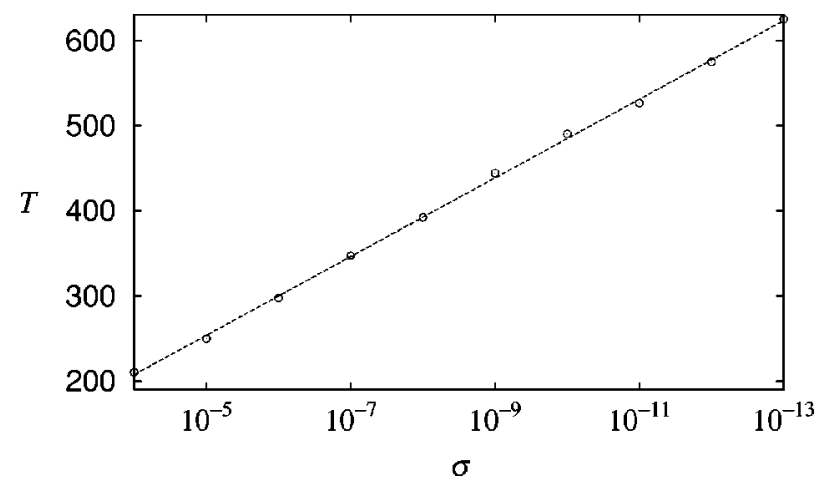

FIG. 13. Switching period vs noise intensity. The line is a linear fitting of the data. 


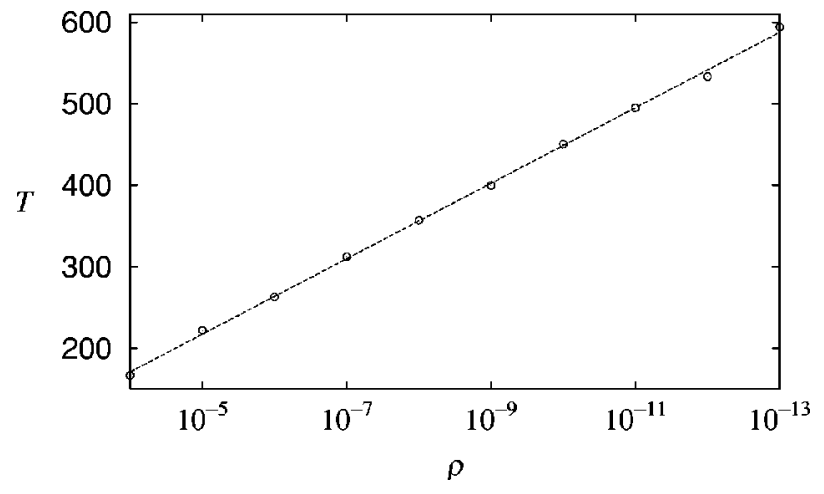

FIG. 14. Switching period vs the square root of the variance of the parameter. The line is a linear fitting of the data.

tion (15). The system is now perturbed slightly so that condition (15) is slightly violated. Assume that a pair of saddles, between which a heteroclinic loop is formed in the symmetric case, still exists. Although a genuine heteroclinic loop could no longer exist in the asymmetric system, the unstable manifold of one saddle will come close to the other saddle. If they are sufficiently close in phase space, the situation is quite similar to the case of applied weak noise, leading to slow switching.

As an example, let $\mu$ in Eq. (16) be Gaussian distributed, with variance $\rho^{2}$. Numerical simulations actually show slow switching without noise. Figure 14, displaying the period versus $\rho$, shows again a logarithmic law. The steepness of $|T / \ln \rho|$ is estimated to be 20 , which implies that the gap $\epsilon$ is now the order of $\rho$. Similar results are obtained when other parameters, e.g., the delay $\tau$, are randomly distributed. We can also break the uniformity in the coupling, and consider a slightly random oscillator network.

\section{CONCLUDING REMARKS}

Slow switching arises when a system with heteroclinic loops is weakly perturbed. The structure of heteroclinic loops in coupled oscillators has been confirmed in the present paper, which is summarized as follows. A pair of unstable twocluster states can be attracting since their unstable manifolds closed into a heteroclinic loop. Such heteroclinic loops are formed stably. Although we commonly consider heteroclinicity structurely unstable, the present heteroclinic loops arise stably in coupled oscillator models, provided that the symmetry condition [Eq. (15)] is satisfied. It can be understood from our argument that the conclusions do not rely so heavily on the specific form of coupled oscillator models. In particular, with delayed coupling, we expect that heteroclinic loops appear in a much broader class of coupled oscillator models. We found that some models, in which unstable solutions had not been considered important, actually form heteroclinic loops by introducing delay in coupling.

As we stated briefly in Sec. VI, heteroclinic loops appear rather easily in an assembly of relaxation oscillators, so that something similar may be expected for homogeneous neuronal assemblies subject to a constant external current. If we associate slow switching with biological rhythms, the property of this phenomenon yields many suggestive ideas. One remarkable feature of slow switching is that a system involved a much longer time scale of dynamics, with which collective order periodically varies, in regard to the intrinsic period of oscillation. This fact is reminiscent of circadian rhythms. One of the intriguing subjects there would be rhythm splitting [14]. A more physiological model should be considered for the study of biological rhythms, and a study in this direction is now in progress.

It may appear that the heteroclinic loop in question is something which could not go beyond some mathematical curiosity, because the symmetry property [Eq. (15)] on which it crucially relies would be more or less violated in real systems. However, the associated phenomenon of slow switching seems to be of much greater physical relevance, because strict symmetry need not be required there. Since noise, heterogeneity, and delay are commonplace in macroscopic systems, some indication of slow switching could well be detected in the real world. In the case of mechanical oscillators, for instance, it would not be difficult to obtain oscillators which are almost identical. Global coupling might also be realized through an electric circuit [15], a vibrating board [16], the surface motion of water [17], and so on. A certain class of surface chemical reactions under oscillatory conditions may provide globally coupled identical oscillators.

The characteristic frequency of slow switching will become shorter when symmetry breaking perturbations become stronger. At the same time, the amplitude of the oscillating order parameter become smaller as the perturbations become stronger. The switching phenomenon is expected to vanish when the strength of the perturbation exceeds a critical value, after which the order parameter of the system becomes stationary. Realistic examples of slow switching, if any, would correspond to the case of strong perturbations. If slow switching survives the strong perturbation, and its frequency becomes comparable to the intrinsic frequency of the oscillators, the dynamics would become even more complicated due to the nonlinear coupling between these modes of motion of comparable time scales. A statistical mechanical approach to this problem would be interesting. As far as we have analyzed, however, slow switching vanishes well before its frequency comes close to the intrinsic frequency. It would also be interesting to find coupled-oscillator models which have more robust structures of slow switching with respect to the symmetry-breaking perturbations.

\section{ACKNOWLEDGMENT}

The authors thank T. Mizuguchi and T. Chawanya for fruitful discussions. They also thank H. Nakao for a careful reading of the manuscript. 
[1] S. Watanabe and S.H. Strogatz, Physica D 74, 197 (1994).

[2] K. Wiesenfeld, P. Colet, and S.H. Strogatz, Phys. Rev. Lett. 76, 404 (1996).

[3] D. Hansel, G. Mato, and C. Meunierm, Europhys. Lett. 23, 367 (1993).

[4] K. Okuda, Physica D 63, 424 (1993).

[5] Y. Kuramoto, Chemical Oscillation, Waves, and Turbulence (Springer, New York, 1984).

[6] R.R. Aliev and V.N. Biktashev, J. Phys. Chem. 98, 9676 (1994).

[7] A.T. Winfree, J. Theor. Biol. 16, 15 (1967).

[8] J. Buck, Q. Rev. Biol. 63, 265 (1988).

[9] K.R. Delaney, A. Gelperin, M.S. Fee, J.A. Flores, R. Gervais, D.W. Tank, and D. Kleinfeld, Proc. Natl. Acad. Sci. U.S.A. 91, 669 (1994).

[10] D. Hansel, G. Mato, and C. Meunier, Phys. Rev. E 48, 3470
(1993).

[11] J.L. Hindmarsh, and R.M. Rose, Proc. R. Soc. London, Ser. B 221, 87 (1984).

[12] S. Raghavachari and J.A. Glazier, Phys. Rev. Lett. 82, 2991 (1999).

[13] L. Abbott and E. Marder, in Method in Neuronal Modeling, edited by C. Koch and I. Segev (MIT Press, Cambridge, MA, 1989).

[14] H. Iglesia, J. Meyer, A. Carpino, and W. Schwartz, Science 290, 799 (2000).

[15] K. Wiesenfeld, P. Colet, and S.H. Strogatz, Phys. Rev. Lett. 76, 404 (1996).

[16] S.H. Strogatz and I. Stewart, Sci. Am. 269, No. 6, 102 (1993).

[17] K. Yoshikawa, N. Oyama, M. Syoji, and S. Nakata, Am. J. Phys. 59, 137 (1991). 